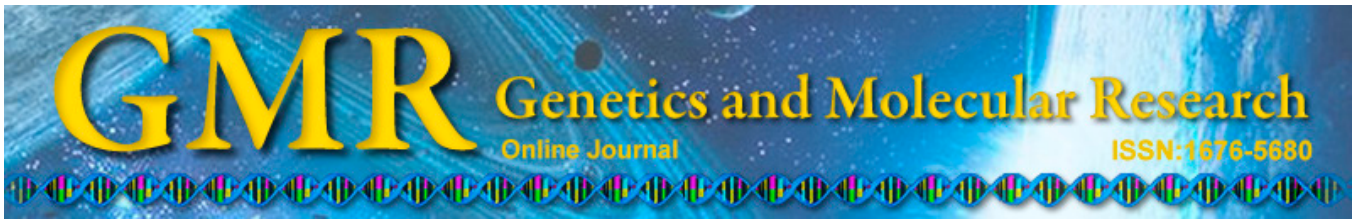

Short Communication

\title{
FYB polymorphisms in Brazilian patients with type I diabetes mellitus and autoimmune polyglandular syndrome type III
}

\author{
C.J.C. Addobbati ${ }^{1,2}$, J. de Azevêdo Silva ${ }^{2}$, N.A.C. Tavares ${ }^{2}$, J. Araújo ${ }^{3}$, \\ R.L. Guimarães ${ }^{1,2}$, L. Brandão ${ }^{1,4}$, S. Crovella ${ }^{1,2}$ and P. Sandrin-Garcia ${ }^{1,2}$ \\ ${ }^{1}$ Departamento de Genética, Universidade Federal de Pernambuco, \\ Recife, PE, Brasil \\ ${ }^{2}$ Laboratório de Imunopatologia Keizo Asami, \\ Universidade Federal de Pernambuco, Recife, PE, Brasil \\ ${ }^{3}$ Serviço de Endocrinologia Pediátrica, Hospital das Clínicas, \\ Universidade Federal de Pernambuco, Recife, PE, Brasil \\ ${ }^{4}$ Departamento de Patologia, Universidade Federal de Pernambuco, \\ Recife, PE, Brasil
}

Corresponding author: C.J.C. Addobbati

E-mail: catarinaaddobbati@hotmail.com

Genet. Mol. Res. 14 (1): 29-33 (2015)

Received July 4, 2014

Accepted October 20, 2014

Published January 15, 2015

DOI http://dx.doi.org/10.4238/2015.January.15.4

\begin{abstract}
The aim of this study was to perform an association study between seven Fyn-binding protein gene (FYB)-tag single nucleotide polymorphisms (SNPs) and type I diabetes mellitus (T1DM), as well as with disease age of onset. We also assessed the role of FYB SNPs in the insurgence of autoimmune polyglandular syndrome type III (APSIII), characterized by the simultaneous presence of autoimmune thyroid disease and celiac disease, in patients with T1DM from a Northeastern Brazilian population. One hundred and seventy-seven patients with T1DM and 190 healthy individuals were genotyped for seven tag SNPs, covering most of the FYB locus, using real-time polymerase chain reaction amplification. There was no significant difference in the distribution of
\end{abstract}


allele and genotype frequencies among patients and healthy individuals. Moreover, none of the tag SNPs were associated either to T1DM age of onset or to the insurgence of APSIII. However, since the FYB protein is a key component in $\mathrm{T}$ cell response, its gene variants might play a role in protein function, which might be testable in a population with different genetic backgrounds or by using functional assays.

Key words: T1DM; Autoimmune polyglandular syndrome type III; Fyn-binding protein; Polymorphisms

\section{INTRODUCTION}

Type I diabetes mellitus (T1DM) is an organ-specific autoimmune disease characterized by the destruction of pancreatic $\beta$ islet cells leading to the impaired production of insulin. The imbalance of the immune system in T1DM patients might lead to autoimmune polyglandular syndrome type III (APSIII), characterized by the simultaneous presence of autoimmune thyroid disease (AITD) and/or celiac disease (CD). The attack by auto-reactive T cells on the pancreas is the main immunological feature in T1DM. Deregulation of T cell homeostasis is a key event in the maintenance of self-tolerance breakdown in T1DM pathogenesis (Dittmar and Kahaly, 2010). The activation of the T cell surface receptor (TCR) triggers intracellular signaling cascades, which result in $\mathrm{T}$ cell activation, differentiation, and acquisition of effector function or apoptosis. Adaptor proteins play a critical role in connecting TCR signals from the cell surface to distal signaling pathways. Alterations in the concentration and function of the adaptor proteins may impair the activation and function of $\mathrm{T}$ lymphocytes (Griffiths et al., 2001; Wang and Rudd, 2008).

The Fyn-binding protein (FYB) is an adaptor protein, also known as adhesion and degranulation promoting adapter protein (ADAP). This protein is involved in T cell signaling activation, and participates in integrin-mediated adhesion, cytoskeletal actin reorganization, and in the stability and duration of cell-cell contact (Griffiths et al., 2001; Wang and Rudd, 2008; Pauker et al., 2011). Since Zou et al. (2008) observed that the loss of FYB leads to defective thymocyte selection and a striking enhancement of T1DM incidence in FYB knockout mice, it could be speculated that this protein might participate in disease establishment. Single nucleotide polymorphisms (SNPs) within the FYB gene (5p13.1) might alter the concentration or function of the protein and, as consequence, contribute to T1DM development. Therefore, in this study we assessed the possible association between seven FYB-tag SNPs (representative SNPs of a group of polymorphisms with high linkage disequilibrium) and the development of T1DM as well as with disease age of onset. In addition, we also assessed the role of FYB polymorphisms in the development of AITD and CD in patients with T1DM from a Northeastern Brazilian population.

\section{MATERIAL AND METHODS}

\section{Subjects}

This study included 177 patients (mean age $13.57 \pm 5$ years; $52 \%$ women and $48 \%$ men; mean age at disease onset $7.57 \pm 4.18$ years) from the three major pediatric endocrinol- 
ogy centers of the Public Health Service from Recife, Brazil (Instituto de Medicina Integral Professor Fernando Figueira, Hospital da Restauração and Hospital das Clínicas - UFPE). The diagnosis of T1DM followed the American Diabetes Association clinical criteria (American Diabetes Association, 2012). Among the 177 patients with T1DM, 10 were diagnosed with CD $\left(\mathrm{AITD}^{-} \mathrm{CD}^{+}\right), 37$ with AITD $\left(\mathrm{AITD}^{+} \mathrm{CD}\right)$, and 4 with both diseases $\left(\mathrm{AITD}^{+} \mathrm{CD}^{+}\right)$. For 3 patients in the T1DM group the information about other autoimmune diseases was missing. The diagnosis for AITD and CD was made as described by Araujo et al. (2009).

The control group comprised 190 healthy individuals (mean age $37.76 \pm 15.08$ years; $85 \%$ women and $15 \%$ men) without family history of autoimmune disorders and recruited from the same geographic area as the patient group. This study was carried out with advanced approval from the Local Ethics Committee (IMIP Numbers: 762/2006 and 1717/2010) and written informed consent was obtained from all participants.

\section{FYB genotyping}

Genomic DNA was isolated from peripheral blood samples using the DNA Wizard Genomic DNA Purification Kit (Promega Madison, WI, USA) following the manufacturer protocol. SNP genotyping was performed with fluorogenic allele-specific probes (Applied Biosystems, Foster City, CA, USA), using an ABI7500 sequence detection system (Applied Biosystems). Seven tag SNPs in the FYB gene (HUGO gene nomenclature Committee ID:4036) were selected from the promoter region to the $3^{\prime}$ untranslated region (UTR) of the gene in order to cover most of the gene locus: rs13188259 [C>T] (promoter region) (probe: C_3195215 20); rs6863066 [C>T] (5'UTR) (probe: C_29184497_10); rs1642515 [G>A] (exon 2) (probe: C_7510102_10); rs379707 [A>C] (exon 15) (probe: C_646794_10); rs2161612 [A>G] (3'UTR) (probe: C_13933712_10); rs358501 [T>C] (3'UTR) (probe: C_646809_10); and rs404122 [A>T] (3'UTR) (probe: C_646808_10) (Figure 1). The tag SNPs were selected by crossing and merging data from the SNPBrowser Software version 4.0 (Applied Biosystems) and the International HapMap Project (www.hapmap.org) as well as the National Center of Biotechnology Information site (NCBI, www.ncbi.nlm.nih.gov). Inclusion criteria were the coverage of a substantial part of the gene and the occurrence of measurable polymorphic variance in both Yoruba in Ibadan, Nigeria (YRI) and Utah residents with Northern and Western European ancestry (CEU) populations.

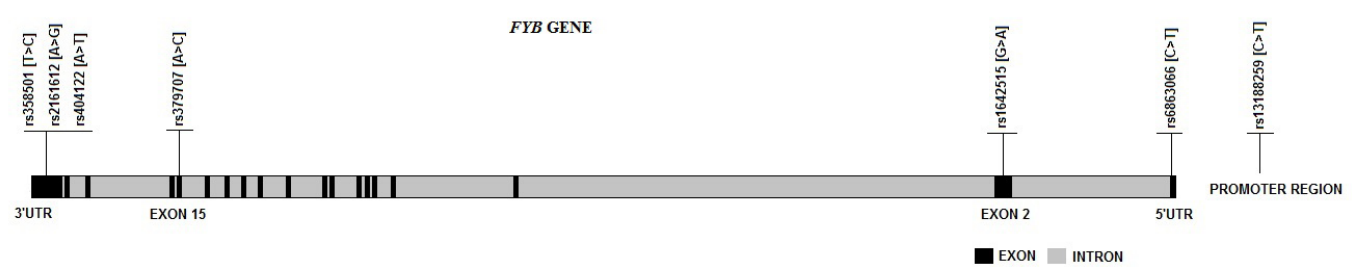

Figure 1. Selected $F Y B$ tag-SNPs. SNP = single nucleotide polymorphism.

\section{Statistical analysis}

Genotype frequencies and Hardy-Weinberg Equilibrium (HWE) were evaluated by the Genotype Transposer software (Cox and Canzian, 2001). The statistical significance of 
differences in allele and genotype frequencies between patients and controls were calculated by Fisher's exact test. The SNPassoc package in the R 3.0.0 software platform (www.r-project. org/) was used to calculate the odds ratios (ORs) and 95\% confidence intervals (95\%CIs). P values $<0.05$ were considered to be statistically significant. The Haploview software (www. broad.mit.edu/mpg/haploview) was used for linkage disequilibrium (LD) analysis in the association of $F Y B$ polymorphisms and T1DM.

\section{RESULTS}

The distribution of allele and genotype frequencies of the seven FYB SNPs studied in patients with T1DM and healthy individuals is depicted in Table 1. No significant differences were observed when comparing the allele and genotype frequencies between patients and controls. When analyzing the frequencies of the tested tag SNPs in APSIII and age at onset, no statistically significant results were found. In addition, no significant association was found between the haplotypes for the seven SNPs and T1DM susceptibility (data not shown).

Table 1. Genotype and allele frequencies of controls and patients with T1DM stratified according to the insurgence of AITD and CD.

\begin{tabular}{|c|c|c|c|c|c|c|c|c|c|c|c|c|}
\hline & & \multicolumn{2}{|c|}{$\mathrm{T} \mathrm{D}^{+} \mathrm{AITD}^{+} \mathrm{CD}^{+}$} & \multicolumn{2}{|c|}{$\mathrm{T}^{1} \mathrm{D}^{+} \mathrm{AITD}^{+} \mathrm{CD}^{-}$} & \multicolumn{2}{|c|}{ T1D $^{+}$AITD-CD $^{+}$} & \multicolumn{2}{|c|}{$\mathrm{T}^{1} \mathrm{D}^{+} \mathrm{AITD}^{-} \mathrm{CD}^{-}$} & \multicolumn{2}{|c|}{$\mathrm{T}_{1} \mathrm{D}^{+}$} & \multirow{2}{*}{$\frac{\text { Controls }}{\mathrm{N}=190(\%}$} \\
\hline & & $\mathrm{N}=4(\%)$ & & $\mathrm{N}=37(\%)$ & & $\mathrm{N}=10(\%)$ & & $\mathrm{N}=123(\%)$ & & $\mathrm{N}=177(\%)$ & & \\
\hline \multirow[t]{5}{*}{ rs13188259 } & $\mathrm{C}$ & $6(75)$ & & $48(65)$ & & $12(60)$ & & $153(62)$ & & $224(63)$ & & $239(63)$ \\
\hline & $\mathrm{T}$ & $2(25)$ & $\mathrm{P}=0.74$ & $26(35)$ & $\mathrm{P}=0.85$ & $8(40)$ & $P=0.98$ & $93(38)$ & $P=0.93$ & $130(37)$ & $P=0.96$ & $141(37)$ \\
\hline & $\mathrm{C} / \mathrm{C}$ & $2(50)$ & & $15(41)$ & & $4(40)$ & & $50(41)$ & & $73(41)$ & & $70(37)$ \\
\hline & $\mathrm{C} / \mathrm{T}$ & $2(50)$ & $\mathrm{P}=0.87$ & $18(49)$ & $P=0.81$ & $4(40)$ & $P=0.91$ & $53(43)$ & $P=0.31$ & $78(44)$ & $P=0.33$ & $99(52)$ \\
\hline & $\mathrm{T} / \mathrm{T}$ & $0(0)$ & $\mathrm{P}=0.93$ & $4(11)$ & $P=0.91$ & $2(20)$ & $\mathrm{P}=0.94$ & $20(16)$ & $P=0.54$ & $26(15)$ & $\mathrm{P}=0.83$ & $21(11)$ \\
\hline \multirow[t]{5}{*}{ rs6863066 } & $\mathrm{C}$ & $6(75)$ & & $50(68)$ & & $12(60)$ & & $160(65)$ & & $233(66)$ & & $250(66)$ \\
\hline & $\mathrm{T}$ & $2(25)$ & $\mathrm{P}=0.87$ & $24(32)$ & $P=0.87$ & $8(40)$ & $P=0.77$ & $86(35)$ & $P=0.92$ & $121(34)$ & $P=0.99$ & $130(34)$ \\
\hline & $\mathrm{C} / \mathrm{C}$ & $2(50)$ & & $16(43)$ & & $4(40)$ & & $52(42)$ & & $76(43)$ & & $82(43)$ \\
\hline & $\mathrm{C} / \mathrm{T}$ & $2(50)$ & $P=0.65$ & $18(49)$ & $P=0.99$ & $4(40)$ & $P=0.77$ & $56(46)$ & $\mathrm{P}=0.99$ & $81(46)$ & $\mathrm{P}=0.93$ & 45) \\
\hline & $\mathrm{T} / \mathrm{T}$ & $0(0)$ & $\mathrm{P}=0.88$ & $3(8)$ & $P=0.82$ & $2(20)$ & $\mathrm{P}=0.85$ & 15 & $P=0.99$ & $20(11)$ & & 12) \\
\hline \multirow[t]{5}{*}{ rs 1642515} & $\mathrm{G}$ & $8(100)$ & & $72(97)$ & & $18(90)$ & & $235(96)$ & & $339(96)$ & & $368(97)$ \\
\hline & $\mathrm{A}$ & $0(0)$ & $\mathrm{P}=0.60$ & $2(3)$ & $P=0.87$ & $2(10)$ & $\mathrm{P}=0.32$ & $11(4)$ & $\mathrm{P}=0.52$ & $15(4)$ & $\mathrm{P}=0.43$ & $12(3)$ \\
\hline & $\mathrm{G} / \mathrm{G}$ & $4(100)$ & & $35(95)$ & & $8(80)$ & & $112(91)$ & & $162(92)$ & & $178(94)$ \\
\hline & $\mathrm{G} / \mathrm{A}$ & $0(0)$. & $P=0.60$ & $2(5)$ & $P=0.87$ & $2(20)$ & $P=0.31$ & $11(9)$ & $\mathrm{P}=0.52$ & $15(8)$ & $\mathrm{P}=0.42$ & $12(6)$ \\
\hline & $\mathrm{A} / \mathrm{A}$ & $0(0)$ & $\mathrm{P}=1$ & $0(0)$ & $P=1$ & $0(0)$ & $P=1$ & $0(0)$ & $P=1$ & $0(0)$ & $\mathrm{P}=1$ & $0(0)$ \\
\hline \multirow[t]{5}{*}{ rs379707 } & A & $2(25)$ & & $48(65)$ & & $13(65)$ & & $135(55)$ & & $202(57)$ & & $213(56)$ \\
\hline & $\mathrm{C}$ & $6(75)$ & $P=0.16$ & $26(35)$ & $P=0.20$ & $7(35)$ & $P=0.58$ & $111(45)$ & $P=0.84$ & $152(43)$ & $P=0.75$ & $167(44)$ \\
\hline & $\mathrm{A} / \mathrm{A}$ & $0(0)$ & & $17(46)$ & & $4(40)$ & & $33(27)$ & & $55(31)$ & & $62(33)$ \\
\hline & $\mathrm{A} / \mathrm{C}$ & $2(50)$ & $\mathrm{P}=0.65$ & $14(38)$ & $\mathrm{P}=0.23$ & $5(50)$ & $\mathrm{P}=0.88$ & $69(56)$ & $\mathrm{P}=0.20$ & $92(52)$ & $\mathrm{P}=0.55$ & $89(46)$ \\
\hline & $\mathrm{C} / \mathrm{C}$ & $2(50)$ & $\mathrm{P}=0.30$ & $6(1$ & $\mathrm{P}=0.37$ & $1(10)$ & $\mathrm{P}=0.71$ & 17) & $\mathrm{P}=0.89$ & $30(17)$ & $P=0.64$ & 21) \\
\hline \multirow[t]{5}{*}{ rs404122 } & A & $6(75)$ & & $52(7$ & & $15(75)$ & & $181(74)$ & & $259(73)$ & & $272(72)$ \\
\hline & $\mathrm{T}$ & $2(25)$ & $\mathrm{P}=0.85$ & $22(30)$ & $\mathrm{P}=0.93$ & $5(25)$ & $\mathrm{P}=0.94$ & 65 & $P=0.65$ & $95(27)$ & $\mathrm{P}=0.54$ & $108(28)$ \\
\hline & $\mathrm{A} / \mathrm{A}$ & $2(50)$ & & $20(54)$ & & $5(50)$ & & $63(51)$ & & $92(52)$ & & $103(54)$ \\
\hline & $\mathrm{A} / \mathrm{T}$ & $2(50)$ & $P=0.75$ & $12(32)$ & $P=0.97$ & $5(50)$ & $P=0.72$ & $55(45)$ & $P=0.25$ & $75(41)$ & $P=0.62$ & $66(35)$ \\
\hline & $\mathrm{T} / \mathrm{T}$ & $0(0)$ & $\mathrm{P}=0.94$ & $5(14)$ & $P=0.94$ & $0(0)$ & $P=0.69$ & $5(4)$ & $P=0.10$ & $10(6)$ & $\mathrm{P}=0.21$ & $21(11)$ \\
\hline \multirow[t]{5}{*}{ rs2161612 } & A & $5(63)$ & & $50(68)$ & & $13(65)$ & & $176(72)$ & & $248(70)$ & & 257 (68) \\
\hline & G & $3(37)$ & $\mathrm{P}=0.94$ & $24(32)$ & $P=0.90$ & $7(35)$ & $P=1$ & $70(28)$ & $P=0.34$ & $106(30)$ & $\mathrm{P}=0.53$ & $123(32)$ \\
\hline & $\mathrm{A} / \mathrm{A}$ & $1(25)$ & & $16(43)$ & & $4(40)$ & & $66(54)$ & & $88(50)$ & & $88(46)$ \\
\hline & $\mathrm{A} / \mathrm{G}$ & $3(75)$ & $\mathrm{P}=0.57$ & $18(49)$ & $P=0.73$ & $5(50)$ & $\mathrm{P}=0.92$ & $44(36)$ & $P=0.24$ & $72(41)$ & $P=0.67$ & $81(43)$ \\
\hline & $\mathrm{G} / \mathrm{G}$ & $0(0)$ & $\mathrm{P}=0.43$ & $3(8)$ & $P=0.97$ & $1(10)$ & $\mathrm{P}=0.59$ & $13(11)$ & $P=0.76$ & $17(10)$ & $\mathrm{P}=0.68$ & $21(11)$ \\
\hline \multirow[t]{5}{*}{ rs 358501} & $\mathrm{~T}$ & $6(75)$ & & $60(81)$ & & $17(85)$ & & $202(82)$ & & $290(82)$ & & $308(81)$ \\
\hline & $\mathrm{C}$ & $2(25)$ & $\mathrm{P}=0.98$ & $14(19)$ & $P=0.87$ & $3(15)$ & $\mathrm{P}=0.88$ & 44 (18) & $P=0.82$ & 64 (18) & $\mathrm{P}=0.84$ & 72 (19) \\
\hline & $\mathrm{T} / \mathrm{T}$ & $2(50)$ & & $24(65)$ & & $7(70)$ & & $80(65)$ & & $115(65)$ & & $128(67)$ \\
\hline & $\mathrm{T} / \mathrm{C}$ & $2(50)$ & $P=0.72$ & $12(32)$ & $P=0.74$ & $3(30)$ & $P=0.78$ & $42(34)$ & $\mathrm{P}=0.37$ & $60(34)$ & $\mathrm{P}=0.11$ & $52(27)$ \\
\hline & $\mathrm{C} / \mathrm{C}$ & $0(0)$ & $\mathrm{P}=0.32$ & $1(3)$ & $\mathrm{P}=0.87$ & $0(0)$ & $\mathrm{P}=0.98$ & $1(1)$ & $\mathrm{P}=0.09$ & $2(1)$ & $\mathrm{P}=0.07$ & $10(6)$ \\
\hline
\end{tabular}

Genotype frequencies were in Hardy Weinberg equilibrium with the exception of rs404122 in controls $(\mathrm{P}=0.0438)$. $\mathrm{T} 1 \mathrm{DM}=$ Type 1 diabetes mellitus; $\mathrm{AITD}=$ autoimmune thyroid disease $\mathrm{CD}=$ celiac disease $\mathrm{P}=\mathrm{P}$ value. 


\section{DISCUSSION}

Even though major pathogenic events in T1DM are known, the complex processes leading to the breakdown of self-tolerance and triggering of disease are yet to be fully elucidated. The study performed by Zou et al. (2008) highlighted some important results indicating that the FYB protein displays a key action in modulating $\mathrm{T}$ cells behavior in non-obese diabetic (NOD) mice. In addition, their results indicated that FYB is present in the pathogenic events in T1DM in murine models. Since $F Y B$ polymorphisms may alter T cell function, we considered the possibility that $F Y B$ SNPs might be related to the susceptibility to T1DM development. Our results indicated that the tag SNPs assessed were not associated with T1DM susceptibility, with AITD and CD development, or with age at onset of T1DM. Our negative association might be related to the fact that, in humans, the self-tolerance breakdown is the result of intricate events not only at the genomic level and therefore not detectable in association studies. Importantly, $F Y B$ is a highly conserved gene with massive intronic sites, and the allele frequencies of $F Y B$ SNPs in the mixed Brazilian population differed from those reported by the HapMap project. Therefore, since this is the first association study involving FYB SNPs and T1DM and APSIII susceptibility, replicate studies in different populations would bring important information about the behavior of $F Y B$ allele and genotype frequencies. In addition, understanding how the variations in this gene might alter the protein function would facilitate the comprehension of the mechanisms in which the FYB protein might participate in T1DM and APSIII pathogenesis.

\section{ACKNOWLEDGMENTS}

Research supported by Coordenação de Aperfeiçoamento de Pessoal de Nível Superior (CAPES), Conselho Nacional de Desenvolvimento Científico e Tecnológico (CNPq), and Fundação de Amparo à Ciência e Tecnologia de Pernambuco (FACEPE).

\section{REFERENCES}

American Diabetes Association (2012). Diagnosis and classification of diabetes mellitus. Diabetes Care 35: S64-S71.

Araujo J, Segat L, Guimarães RL, Brandao LA, et al. (2009). Mannose binding lectin gene polymorphisms and associated auto-immune diseases in type 1 diabetes Brazilian patients. Clin. Immunol. 131: 254-259.

Cox DG and Canzian F (2001). Genotype transposer: automated genotype manipulation for linkage disequilibrium analysis. Bioinformatics 17: 738-739.

Dittmar M and Kahaly GJ (2010). Genetics of the autoimmune polyglandular syndrome type 3 variant. Thyroid 20: 737743.

Griffiths EK, Krawczyk C, Kong YY, Raab M, et al. (2001). Positive regulation of T cell activation and integrin adhesion by the adapter Fyb/Slap. Science 293: 2260-2263.

Pauker MH, Reicher B, Fried S, Perl O, et al. (2011). Functional cooperation between the proteins Nck and ADAP is fundamental for actin reorganization. Mol. Cell Biol. 31: 2653-2666.

Wang H and Rudd CE (2008). SKAP-55, SKAP-55-related and ADAP adaptors modulate integrin-mediated immune-cell adhesion. Trends Cell Biol. 18: 486-493.

Zou L, Mendez F, Martin-Orozco N and Peterson EJ (2008). Defective positive selection results in T cell lymphopenia and increased autoimmune diabetes in ADAP-deficient BDC2.5-C57BL/6 mice. Eur. J. Immunol. 38: 986-994. 